\title{
Cost-Utility Analysis of Dapagliflozin as an Add-On to Standard Treatment for Patients with Type 2 Diabetes and High Risk of Cardiovascular Disease in Thailand
}

\author{
Chaicharn Deerochanawong · Kriengsak Vareesangthip • \\ Dilok Piyayotai $\cdot$ Dittaya Thongsuk $\cdot$ Nuch Pojchaijongdee $\cdot$ \\ Unchalee Permsuwan (1)
}

Received: April 24, 2021 / Accepted: May 24, 2021 / Published online: June 9, 2021

(c) The Author(s) 2021

\section{ABSTRACT}

Introduction: Diabetes treatment has incurred financial burden. We examined the cost-utility of adding dapagliflozin to the standard treatment for treating type 2 diabetes (T2DM) with cardiovascular risk in a Thai context.

Supplementary Information The online version contains supplementary material available at https:// doi.org/10.1007/s13300-021-01088-w.

C. Deerochanawong

Rajavithi Hospital, College of Medicine, Rangsit University, Ministry of Public Health, Bangkok 10400, Thailand

K. Vareesangthip

Renal Division, Department of Medicine, Faculty of Medicine Siriraj Hospital, Mahidol University, Bangkok 10700, Thailand

D. Piyayotai

Department of Medicine, Faculty of Medicine, Thammasat University, Pathum Thani 12120, Thailand

D. Thongsuk · N. Pojchaijongdee

AstraZeneca (Thailand) Ltd., Bangkok 10120

Thailand

U. Permsuwan $(\bowtie)$

Department of Pharmaceutical Care, Faculty of Pharmacy, Chiang Mai University, Chiang Mai 50200, Thailand

e-mail: unchalee.permsuwan@cmu.ac.th
Methods: A two-part model, decision tree and Markov models, was developed to capture the benefits in terms of heart failure (HF) and chronic kidney disease. The model was used to estimate the lifetime costs and outcomes from a societal perspective. Costs were based on local data while the transitional probabilities and utilities were derived from the DECLARETIMI 58 clinical trial and published studies. Future costs and outcomes were discounted at an annual rate of $3 \%$. The results were reported as incremental cost-effectiveness ratios (ICER). One-way and probabilistic sensitivity analyses were performed to investigate parameter uncertainty.

Results: The increased cost of adding dapagliflozin from 8707 USD to 14,455 USD was associated with an increase in quality-adjusted life years (QALYs) from 9.28 to 9.58, yielding an ICER of 18,988 USD/QALY. Compared with the standard treatment, the dapagliflozin group acquired more clinical benefits in terms of fewer HF hospitalizations and macroalbuminuria. Sensitivity analyses revealed that with high prevalence of diabetic nephropathy of $29.4-43.9 \%$, the ICER would decline to 5591-8014 USD/QALY.

Conclusion: On the basis of the DECLARE study with low incidence of T2DM complications and 4.2 years of median follow-up duration, the add-on dapagliflozin results in an ICER of 18,988 USD/QALY, which exceeds the local threshold of 5310 USD/QALY. Dapagliflozin 
would show better value for money in the context of high prevalence of T2DM complications.

Keywords: Cardiovascular disease; Chronic kidney disease; Dapagliflozin; Diabetes; SGLT2 inhibitor

\section{Key Summary Points}

Use of dapagliflozin may be a costeffective add-on strategy in type 2 diabetes with high cardiovascular risk.

Although drug costs were high, dapagliflozin shows clinical benefits in terms of heart failure and chronic kidney disease.

Base-case incremental cost-effectiveness ratio exceeds the local threshold in Thailand.

With the real high prevalence of chronic kidney disease in Thailand, add-on dapagliflozin shows better value for money.

\section{DIGITAL FEATURES}

This article is published with digital features, including a summary slide, to facilitate understanding of the article. To view digital features for this article go to https://doi.org/10.6084/ m9.figshare.14626524.

\section{INTRODUCTION}

Type 2 diabetes mellitus (T2DM) is a growing public health issue globally. In 2019, this condition affected around 463 million people worldwide and the prevalence is increasing over time [1]. T2DM, especially with poor glycemic control, is strongly associated with a risk of micro- and macrovascular complications and mortality [2]. These comorbidities place substantial cost burden on the healthcare system. In Thailand, approximately $44 \%$ of outpatients with T2DM reported a history of microvascular complications [3], 16\% reported a history of cardiovascular disease, and $5 \%$ had heart failure [4].

About half of the direct medical cost was allocated to hospital care while the cost of medicine was only $14 \%$ and the cost of treating a patient with diabetes increased exponentially when the patient developed complications [5].

Clinical guidelines recognize the importance of considering management of patients with T2DM beyond glycemic control by counting risks and complications [6]. Sodium-glucose cotransporter 2 (SGLT2) inhibitors and glucagonlike peptide 1 (GLP-1) receptor agonists are considered effective add-on treatments for patients with T2DM and additional risk factors such as increased cardiovascular and/or renal risk [6]. The SGLT2 inhibitors demonstrate robust benefits on reducing hospitalization for heart failure (HHF) and slowing progression of renal disease and have shown a modest benefit on atherosclerotic major adverse cardiovascular events (MACE), which seems narrowed to patients with established atherosclerotic cardiovascular disease (ASCVD) [7].

The Dapagliflozin Effect on Cardiovascular Events-Thrombolysis in Myocardial Infarction 58 (DECLARE TIMI-58) is a large cardiovascular outcome trial of dapagliflozin compared to placebo as an add-on treatment to the standard treatment, including over 17,000 patients with T2DM and established cardiovascular disease (CVD) or multiple risk factors for CVD [8]. Over the follow-up period of 4.2 years, dapagliflozin demonstrated a significant reduction in cardiovascular $(\mathrm{CV})$ death/hospitalized heart failure (HHF), and HHF alone in a broad patient population, and was superior to placebo when added to standard care. The reduction in HHF was consistent across patients with multiple risk factors and established CVD, suggesting that use in T2DM before established CV disease may be beneficial. The data also suggested beneficial effects on renal function associated with dapagliflozin, demonstrated by a reduction in the renal composite endpoint (at least $40 \%$ decrease in estimated glomerular filtration rate [eGFR] to 
below $60 \mathrm{ml} / \mathrm{min} / 1.73 \mathrm{~m}^{2}$, end-stage renal disease (ESRD), or renal or CV death). These clinical benefits of dapagliflozin could consequently reduce healthcare spending on costly long-term treatment for cardiorenal complications such as cardiovascular intervention, hospital admission, dialysis, and renal replacement therapy. However, there is no local economic evidence based on the cardiorenal protection benefits from the DECLARE TIMI-58 study. Hence, this study was conducted to analyze the cost-utility of adding dapagliflozin to the standard treatment for treating patients with type 2 diabetes and high cardiovascular risk in a Thai context.

\section{METHODS}

\section{Cohort Population}

The modelled population is assumed to be of equivalent characteristics to the patients enrolled in the DECLARE-TIMI 58 clinical trial [8]. In brief, the eligible patients were at least 40 years of age, having type 2 diabetes with $\mathrm{HbA1c}$ in a range of $6.5-12 \%$ and a creatinine clearance of at least $60 \mathrm{ml} / \mathrm{min}$, and having multiple risk factors or established ASCVD. Patients in the DECLARE study were followed for a median of 4.2 years.

\section{Intervention and Comparator}

The intervention group in this study was patients who received $10 \mathrm{mg}$ of dapagliflozin daily as an add-on to standard treatment. On the basis of the DECLARE-TIMI 58 clinical trial [8], the standard treatment would be insulin, metformin, sulfonylurea, dipeptidyl peptidase 4 (DPP4) inhibitors, and GLP-1 receptor agonist. Patients in the dapagliflozin groups received the same proportion of the aforementioned glucose-lowering therapies as those in the standard treatment.

\section{Model Structure}

A two-part model was developed on the basis of the clinical evidence from the DECLARE-
TIMI 58 clinical trial [8]. The first component is a decision tree model (Fig. S1) followed by the lifetime Markov models. The Markov models were divided into the diabetes Markov model (DM Markov model) (Fig. S2), the heart failure Markov model (HF Markov model) (Fig. S3), and the chronic kidney disease Markov model (CKD Markov model) (Fig. S4). All figures are shown in the supplementary material.

The cohort population was entered into the decision tree model. The cohort might or might not have experienced an HF event depending on the probability from the DECLARE-TIMI 58 clinical trial [8]. Patients with HF would subsequently be moved to the HF Markov model. Patients with no HF might possibly develop CKD later on the basis of the probability from the DECLARE-TIMI 58 clinical trial [8]. We assumed that no patients encountered both HF and CKD in this study. Patients who had a CKD event would be moved to the CKD Markov model. Patients without CKD would be moved to the DM Markov model.

The DM Markov model comprises two health states: T2DM and death. Patients without HF or CKD would be moved to the DM Markov model starting at the T2DM health state. When time passed, patients would still be alive at the T2DM health state or move to death state. The cycle length was 1 year with lifetime horizon.

We used the HF Markov model based on a previous HF study in Thailand [9]. Basically, the HF Markov model comprises three health states: stable HF, hospitalization, and death. Death state is separated into CV death and non-cardiovascular death (non-CV death). Patients with HF would be entered into the HF Markov model at the state of hospitalization. Those hospitalized patients with HF could remain in the same health state, or improve and move back to the stable HF health state, or get worse and die with $\mathrm{CV}$ cause or other causes. To capture the hospitalization consequences from the HF event, a three-cycle length was used in this Markov model and eventually reported the annual cost and outcome, similar to the CKD and DM Markov models.

We developed the CKD Markov model based on the literature review [10]. The CKD Markov model comprises six health states: 
normoalbuminuria, microalbuminuria, macroalbuminuria, elevated serum creatinine, end-stage renal disease (dialysis and transplantation), and death. Patients would enter the Markov model at the state of normoalbuminuria and subsequently be moved to other health states on the basis of transitional probabilities. Finally, all patients would enter the absorbing health state, which is the death state.

\section{Input Parameters}

\section{Transitional Probabilities Used in Long-Term DM Markov Model}

The increased excess risk of death has been noted in patients with T2DM [11]. The adjusted hazard ratio (HR) for death from any cause in patients with T2DM aged 55-64 years was 1.79 (95\% CI 1.73-1.85). To estimate the mortality rate of patients with T2DM in Thailand, the agespecific mortality rate of the Thai population, with data from the Ministry of Public Health [12] and the burden of disease in the Thai population [13], was multiplied by this HR. Next, we converted rate to risk using the formula $p=1-\exp (-r t)$, where $p$ is probability, $r$ is rate, and $t$ is duration.

On the basis of the findings from the DECLARE-TIMI 58 clinical trial [8], patients in the dapagliflozin group had an HR of 0.93 (95\% CI 0.82-1.04) compared to the standard group. Therefore, we adjusted the age-specific mortality rate of the Thai T2DM population mentioned above by 0.93 for the dapagliflozin group.

\section{Transitional Probabilities Used in the Long- Term HF Markov Model}

We obtained the transitional probability of HF hospitalization from the DECLARE-TIMI 58 clinical trial [8]. The event rates were 6.2 vs 8.5 per 1000 patient-year for the dapagliflozin and standard groups, respectively. These rates were converted to 3-month probabilities. Hospitalized patients with HF can be readmitted for any cause within 30 days. As a result of no readmission data being reported from the DECLARETIMI 58 clinical trial [8], we used a $34 \%$ rate of readmission from the Thai HF national database
[14]. This rate was converted into a risk of 0.2882 .

Death from cardiovascular cause was not significantly different between the dapagliflozin and standard groups (7.0 vs 7.1 per 1000 patient-year). These rates were converted to 3 -month probabilities of 0.0017 vs 0.0018 , respectively. For the HF Markov model, we separated all CV deaths into either hospitalized or non-hospitalized CV deaths. From a total of 201,709 hospitalized HF elderly patients in Thailand, $17.6 \%$ were reported as $\mathrm{CV}$ deaths [15]. We used this figure to derive the risk of CV death in hospital for both the dapagliflozin and the standard treatment groups. As a result of the small difference in CV deaths for both groups, the 3-month risk of hospitalized CV death for the dapagliflozin group was slightly lower than the standard group (0.000308 vs 0.000312, respectively). The risk of non-hospitalized CV deaths was calculated by subtracting all hospitalized CV deaths from CV deaths, which was equal to 0.0014 for the dapagliflozin group and 0.0015 for the standard treatment group.

Hospitalized non-CV deaths were obtained from the DECLARE-TIMI 58 clinical trial [8]. The dapagliflozin group had fewer deaths from non-cardiovascular causes than the standard treatment group (6.0 vs 6.8 per 1000 patientyear). These rates were then converted into 3 -month risks (0.0015 vs 0.0017 respectively). For non-CV deaths outside hospital, the agespecific mortality rate of the Thai T2DM population with $\mathrm{CV}$ deaths removed was used in the model.

\section{Transitional Probabilities Used in Long-Term CKD Markov Model}

On the basis of the findings from the DECLARETIMI 58 clinical trial [8], dapagliflozin decreases albuminuria progression and increases albuminuria regression. Therefore, the transition probabilities of dapagliflozin and standard treatment groups from normoalbuminuria to microalbuminuria, microalbuminuria back to normoalbuminuria, normoalbuminuria to macroalbuminuria, macroalbuminuria back to normoalbuminuria, microalbuminuria to macroalbuminuria, and macroalbuminuria back to microalbuminuria were based on the 
Table 1 Transitional probabilities

\begin{tabular}{|c|c|c|c|}
\hline Parameter & Value & Range & References \\
\hline \multicolumn{4}{|l|}{ Short-term decision tree model } \\
\hline \multicolumn{4}{|l|}{ Dapagliflozin } \\
\hline $\mathrm{HF}$ & 0.0247 & $0.0230-0.0264$ & \multirow[t]{2}{*}{ Wiviott $[8]$} \\
\hline $\mathrm{CKD}$ & 0.0148 & $0.0135-0.0161$ & \\
\hline \multicolumn{4}{|l|}{ Standard treatment } \\
\hline $\mathrm{HF}$ & 0.0333 & $0.0314-0.0353$ & \multirow[t]{2}{*}{ Wiviott $[8]$} \\
\hline CKD & 0.0277 & $0.0260-0.0295$ & \\
\hline \multicolumn{4}{|l|}{ Long-term DM Markov model } \\
\hline Age-specific mortality rate of Thai population & & & \multirow{7}{*}{$\begin{array}{l}\text { Ministry of Public Health [12], } \\
\text { burden of disease in Thai population [13 }\end{array}$} \\
\hline $60-64$ & 0.0142 & & \\
\hline $65-69$ & 0.0213 & & \\
\hline $70-74$ & 0.0325 & & \\
\hline $75-79$ & 0.0517 & & \\
\hline $80-84$ & 0.0822 & & \\
\hline $85-89$ and above & 0.1457 & & \\
\hline HR of DM mortality & 1.79 & $1.73-1.85$ & Tancredi $[11]$ \\
\hline HR of death from any cause (dapagliflozin vs placebo) & 0.93 & $0.82-1.04$ & Wiviott $[8]$ \\
\hline \multicolumn{4}{|l|}{ Long-term HF Markov model (3-month cycle) } \\
\hline \multicolumn{4}{|l|}{ Dapagliflozin } \\
\hline HF hospitalization & $0.0015^{\mathrm{a}}$ & $0.0014-0.0017$ & Wiviott $[8]$ \\
\hline Hospitalized cardiovascular death & $0.0003^{\mathrm{b}}$ & $0.0003-0.0003$ & Wiviott [8], Krittayaphong [15] \\
\hline Hospitalized non-cardiovascular death & $0.0015^{\mathrm{c}}$ & $0.0013-0.0016$ & Wiviott $[8]$ \\
\hline Non-hospitalized cardiovascular death & $0.0014^{\mathrm{d}}$ & $0.0013-0.0016$ & Wiviott $[8]$ \\
\hline \multicolumn{4}{|l|}{ Standard treatment } \\
\hline HF hospitalization & $0.0021^{\mathrm{e}}$ & $0.0019-0.0023$ & Wiviott $[8]$ \\
\hline Hospitalized cardiovascular death & $0.0003^{\mathrm{f}}$ & $0.0003-0.0003$ & Wiviott [8], Krittayaphong [15] \\
\hline Hospitalized non-cardiovascular death & $0.0017^{\mathrm{g}}$ & $0.0015-0.0019$ & Wiviott $[8]$ \\
\hline Non-hospitalized cardiovascular death & $0.0015^{\mathrm{h}}$ & $0.0013-0.0016$ & Wiviott $[8]$ \\
\hline \multicolumn{4}{|l|}{ Dapagliflozin and standard treatment } \\
\hline 30-day readmission & 0.2882 & $0.2594-0.3171$ & Janwanishstaporn [14] \\
\hline \multicolumn{4}{|l|}{ Long-term CKD Markov model } \\
\hline Dapagliflozin & & & \\
\hline
\end{tabular}


Table 1 continued

\begin{tabular}{|c|c|c|c|}
\hline Parameter & Value & Range & References \\
\hline Normoalbuminuria to microalbuminuria & $0.0252^{\mathrm{i}}$ & $0.0227-0.0277$ & Mosenzon [16] \\
\hline Normoalbuminuria to macroalbuminuria & $0.0075^{j}$ & $0.0067-0.0082$ & \\
\hline Microalbuminuria to normoalbuminuria & $0.1089^{k}$ & $0.0980-0.1198$ & \\
\hline Microalbuminuria to macroalbuminuria & $0.1365^{1}$ & $0.1228-0.1501$ & \\
\hline Macroalbuminuria to normoalbuminuria & $0.0144^{\mathrm{m}}$ & $0.0129-0.0158$ & \\
\hline Macroalbuminuria to microalbuminuria & $0.1201^{\mathrm{n}}$ & $0.1081-0.1322$ & \\
\hline Elevated SCr to ESRD & $0.0457^{\circ}$ & $0.0411-0.0502$ & \\
\hline \multicolumn{4}{|l|}{ Standard treatment } \\
\hline Normoalbuminuria to microalbuminuria & $0.0268^{\mathrm{P}}$ & $0.0242-0.0295$ & Mosenzon [16] \\
\hline Normoalbuminuria to macroalbuminuria & $0.0138^{\mathrm{q}}$ & $0.0124-0.0152$ & \\
\hline Microalbuminuria to normoalbuminuria & $0.0771^{\mathrm{r}}$ & $0.0694-0.0848$ & \\
\hline Microalbuminuria to macroalbuminuria & $0.2045^{\mathrm{s}}$ & $0.1841-0.2250$ & \\
\hline Macroalbuminuria to normoalbuminuria & $0.0118^{\mathrm{t}}$ & $0.0106-0.0130$ & \\
\hline Macroalbuminuria to microalbuminuria & $0.0679^{\mathrm{u}}$ & $0.0611-0.0747$ & \\
\hline Elevated SCr to ESRD & 0.1400 & $0.1260-0.1540$ & Srisubat [17] \\
\hline \multicolumn{4}{|l|}{ Dapagliflozin and standard treatment } \\
\hline Normoalbuminuria to elevated $\mathrm{SCr}$ & 0.0010 & $0.0009-0.0011$ & Adler [18] \\
\hline Normoalbuminuria to death & 0.0260 & $0.0234-0.0286$ & Sugrue [10] \\
\hline Microalbuminuria to elevated SCr & 0.0030 & $0.0027-0.0033$ & Adler [18] \\
\hline Microalbuminuria to death & 0.0380 & $0.0342-0.0418$ & Sugrue [10] \\
\hline Macroalbuminuria to elevated $\mathrm{SCr}$ & 0.0230 & $0.0207-0.0253$ & Adler [18] \\
\hline Macroalbuminuria to death & 0.1010 & $0.0909-0.1111$ & Sugrue [10] \\
\hline Elevated SCr to death & 0.1920 & $0.1728-0.2112$ & Adler [18] \\
\hline
\end{tabular}


Table 1 continued

\begin{tabular}{llll}
\hline Parameter & Value & Range & References \\
\hline Dialysis to transplantation & 0.0550 & $0.0495-0.0605$ & Sugrue [10] \\
Dialysis to death & 0.1770 & $0.1593-0.1947$ & \\
Transplantation to dialysis & 0.0820 & $0.0738-0.0902$ & \\
Transplantation to death & 0.0530 & $0.0477-0.0583$ & \\
\hline
\end{tabular}

$C K D$ chronic kidney disease, $C V$ cardiovascular, $D M$ diabetes, $H F$ heart failure, $H R$ hazard ratio, ESRD end stage renal disease, $S C r$ serum creatinine

${ }^{\mathrm{a}}$ HF hospitalization for dapagliflozin: 1-year rate $=6.2 / 1000=0.0062$. 3-month probability $=1-\exp (-0.0062 / 4)=0.0015$

b Hospitalized CV death for dapagliflozin: 1-year rate of CV death from DECLARE $=7.0 / 1000=0.0070$. 1-year rate of hospitalized CV death in Thailand $=17.6 \%$. 3-month rate of hospitalized $\mathrm{CV}$ death $=(17.6 \% \times 0.007) / 4=0.0003$. 3-month probability of hospitalized $\mathrm{CV}$ death $=1-\exp (-0.0003)=0.0003$

${ }^{c}$ Hospitalized non-CV death for dapagliflozin: 1-year rate of non-CV death from DECLARE $=6.0 / 1000=0.0060$. 3-month probability of hospitalized $\mathrm{CV}$ death $=1-\exp (-0.0060 / 4)=0.0015$

${ }^{\mathrm{d}}$ Non-hospitalized CV death for dapagliflozin: 3-month probability of CV death $=1-\exp (-0.0070 / 4)=0.0017$. 3-month probability of nonhospitalized CV death $=0.0017-0.0003=0.0014$

${ }^{\mathrm{e}}$ HF hospitalization for standard treatment: 1 -year rate $=8.5 / 1000=0.0085 .3$-month probability $=1-\exp (-0.0085 / 4)=0.0021$

${ }^{\mathrm{f}}$ Hospitalized CV death for standard treatment: 1 -year rate of CV death from DECLARE $=7.1 / 1000=0.0071$. 3-month rate of hospitalized CV death $=(17.6 \% \times 0.0071) / 4=0.0003$. 3-month probability of hospitalized CV death $=1-\exp (-0.0003)=0.0003$

${ }^{\mathrm{g}}$ Hospitalized non-CV death for standard treatment: 1-year rate of non-CV death from DECLARE $=6.8 / 1000=0.0068$. 3-month probability of hospitalized CV death $=1-\exp (-0.0068 / 4)=0.0017$

${ }^{\text {h }}$ Non-hospitalized CV death for standard treatment: 3-month probability of CV death $=1-\exp (-0.0071 / 4)=0.0018$. 3-month probability of nonhospitalized CV death $=0.0018-0.0003=0.0015$

${ }^{\mathrm{i}}$ Normoalbuminuria to microalbuminuria for dapagliflozin: probability $=591 / 5819=0.102$ in 4.2 years. 1-year rate $=-(\ln (1-0.102)) / 4.2=0.025$. 1 -year probability $=1-\exp (-0.025)=0.0252$

${ }^{j}$ Normoalbuminuria to macroalbuminuria for dapagliflozin: probability $=181 / 5819=0.031$ in 4.2 years. 1-year rate $=-(\ln (1-0.031)) / 4.2=0.008$. 1 -year probability $=1-\exp (-0.008)=0.0075$

${ }^{\mathrm{k}}$ Microalbuminuria to normoalbuminuria for dapagliflozin: probability $=774 / 2017=0.384$ in 4.2 years. 1-year rate $=-(\ln (1-0.384)) / 4.2=0.115$. 1 -year probability $=1-\exp (-0.115)=0.1089$

${ }^{1}$ Microalbuminuria to macroalbuminuria for dapagliflozin: probability $=928 / 2017=0.460$ in 4.2 years. 1 -year rate $=-(\ln (1-0.460)) / 4.2=0.147$. 1 -year probability $=1-\exp (-0.147)=0.1365$

$\mathrm{m}$ Macroalbuminuria to normoalbuminuria for dapagliflozin: probability $=35 / 594=0.059$ in 4.2 years. 1-year rate $=-(\ln (1-0.059)) / 4.2=0.014$. 1 -year probability $=1-\exp (-0.014)=0.0144$

${ }^{\mathrm{n}}$ Macroalbuminuria to microalbuminuria for dapagliflozin: probability $=247 / 594=0.416$ in 4.2 years. 1 -year rate $=-(\ln (1-0.416)) / 4.2=0.128$. 1 -year probability $=1-\exp (-0.128)=0.1201$

${ }^{\circ}$ Elevated serum creatinine to ESRD for dapagliflozin: $\mathrm{HR}=0.31$. 1-year rate for standard treatment $=-\ln (1-0.140)=0.151$. 1 -year rate for dapagliflozin $=0.151 \times 0.31=0.047 .1$-year probability $=1-\exp (-0.047)=0.0457$

P Normoalbuminuria to microalbuminuria for standard treatment: probability $=629 / 5825=0.108$ in 4.2 years. 1 -year rate $=-(\ln (1-0.108))$ / $4.2=0.027$. 1-year probability $=1-\exp (-0.027)=0.0268$

q Normoalbuminuria to macroalbuminuria for standard treatment: probability $=330 / 5825=0.057$ in 4.2 years. 1 -year rate $=-(\ln (1-0.057))$ / $4.2=0.014$. 1-year probability $=1-\exp (-0.014)=0.0138$

${ }^{\mathrm{r}}$ Microalbuminuria to normoalbuminuria for standard treatment: probability $=576 / 2013=0.286$ in 4.2 years. 1 -year rate $=-(\ln (1-0.286)) /$ $4.2=0.080 .1$-year probability $=1-\exp (-0.080)=0.0771$

${ }^{s}$ Microalbuminuria to macroalbuminuria for standard treatment: probability $=1243 / 2013=0.617$ in 4.2 years. 1 -year rate $=-(\ln (1-0.617))$ / $4.2=0.229 .1$-year probability $=1-\exp (-0.229)=0.2045$

${ }^{t}$ Macroalbuminuria to normoalbuminuria for standard treatment: probability $=28 / 575=0.049$ in 4.2 years. 1 -year rate $=-(\ln (1-0.049))$ / $4.2=0.012$. 1-year probability $=1-\exp (-0.012)=0.0118$

"Macroalbuminuria to microalbuminuria for standard treatment: probability $=147 / 575=0.256$ in 4.2 years. 1 -year rate $=-(\ln (1-0.256))$ / $4.2=0.070 .1$-year probability $=1-\exp (-0.070)=0.0679$ 
Table 2 Cost and utility inputs

\begin{tabular}{|c|c|c|c|}
\hline Item & Value & Range & References \\
\hline \multicolumn{4}{|l|}{ Costs } \\
\hline \multicolumn{4}{|l|}{ Heart failure (per event) } \\
\hline Heart failure hospitalization & 88,313 & $70,650-105,976$ & Deerochanawong [20] \\
\hline \multicolumn{4}{|l|}{ Diabetes (per year) } \\
\hline Diabetes treatment & 21,240 & $16,992-25,488$ & Riewpaiboon [21] \\
\hline \multicolumn{4}{|l|}{ Chronic kidney disease (per year) } \\
\hline Predialysis & 85,478 & $68,382-102,573$ & Deerochanawong [20] \\
\hline Dialysis set up & $32,681^{a}$ & $26,145-39,217$ & Teerawattananon $[22]$ \\
\hline Dialysis & 557,623 & $446,098-669,147$ & \\
\hline Transplantation & 379,021 & $303,217-454,826$ & NHSO [23] \\
\hline Follow-up after transplantation (first 2 years) & 47,500 & $38,000-57,000$ & \\
\hline Follow-up after transplantation (third year and onward) & 15,000 & $12,000-18,000$ & \\
\hline \multicolumn{4}{|l|}{ Drug (per year) } \\
\hline Dapagliflozin & 14,638 & $11,710-17,565$ & DMSIC [24] \\
\hline \multicolumn{4}{|l|}{ Adverse events ${ }^{\mathrm{b}}$ (per event) } \\
\hline Major hypoglycemic event & 51,607 & $2346-100,867$ & Siriraj hospital database [25] \\
\hline Diabetic ketoacidosis & 64,763 & $14,388-115,138$ & \\
\hline Acute kidney injury & 110,906 & $95,051-316,864$ & \\
\hline Genital infection & 11 & & DMSIC [24] \\
\hline \multicolumn{4}{|l|}{ Direct non-medical cost } \\
\hline \multicolumn{4}{|l|}{ Heart failure and diabetes ${ }^{c}$ (per event) } \\
\hline Transportation & 155 & $143-168$ & Standard cost list [26] \\
\hline Food & 57 & $51-63$ & \\
\hline \multicolumn{4}{|l|}{ Chronic kidney disease (per year) } \\
\hline Normoalbuminuria & 1045 & $836-1254$ & Srisubat $[17]$ \\
\hline Microalbuminuria & 1400 & $1120-1680$ & \\
\hline Macroalbuminuria & 2116 & $1693-2540$ & \\
\hline Dialysis & 8170 & $6536-9804$ & \\
\hline \multicolumn{4}{|l|}{ Utilities } \\
\hline \multicolumn{4}{|l|}{ Heart failure } \\
\hline Hospitalization & 0.65 & $0.55-0.75$ & Adena [37] \\
\hline Stable & 0.75 & $0.65-0.85$ & \\
\hline
\end{tabular}


Table 2 continued

\begin{tabular}{llll}
\hline Item & Value & Range & References \\
\hline Diabetes & & & \\
Diabetes & 0.814 & $0.733-0.895$ & Clarke [38] \\
Chronic kidney disease & & & \\
Normoalbuminuria & 0.72 & $0.696-0.744$ & Srisubat [17] \\
Microalbuminuria & 0.72 & $0.696-0.744$ & \\
Macroalbuminuria & 0.59 & $0.549-0.631$ & \\
Dialysis & 0.55 & $0.499-0.601$ & \\
Transplantation & 0.83 & $0.747-0.913$ & Li [39] \\
\hline
\end{tabular}

DMSIC Drug and Medical Supply Information Center, NHSO the National Health Security Office

${ }^{a}$ Average on the proportion of peritoneal dialysis (30\%) and hemodialysis (70\%)

${ }^{\mathrm{b}}$ Cost of adverse event treatment $=$ cost per event $\times$ prevalence of such adverse event

c Patients visit a hospital 4 times per year. Direct non-medical cost per year $=$ cost per event $\times 4$

DECLARE-TIMI 58 clinical trial [8]. The detail of how to derive the probability is shown in the footnote of Table 1. Other transition probabilities were based on the literature review $[10,11,14-18]$. All transition probabilities are shown in Table 1.

\section{Costs}

The Thai Health Technology Assessment (HTA) guideline [19] recommends a societal perspective for cost-utility study. Hence, direct medical costs and direct non-medical costs were included in this study. Indirect costs, such as productivity loss, or time loss was not taken into consideration in order to avoid double counting when time loss is measured as an outcome in terms of quality-adjusted life years [19]. In general, cost data were based on costs in Thailand and were obtained from several reliable resources. The costs of diabetes treatment, heart failure hospitalization, and dialysis were obtained from published Thai studies $[17,20-26]$. The costs of transplantation and follow-up were from the reimbursement list of the universal health coverage scheme. The drug cost was from the Drug and Medical Supply Information Center, Ministry of Public Health. The costs of adverse events were from the cost- effectiveness study of SGLT2 inhibitors for economic evidence to justify whether SGLT2 inhibitors should be listed in the National List of Essential Medicine in Thailand [25]. Direct nonmedical costs were from the literature review and standard cost list in Thailand. All costs were inflated with the consumer price index in the medical care section [27], and presented in 2019 . The costs were converted at a rate of 30.13 THB per USD as of 28 December 2020 [28].

\section{Utilities}

The utilities of patients with T2DM and CKD were obtained from the cost-effectiveness study of Thai diabetics [17], which reported the impact of worsening CKD stages on the quality of life of patients with T2DM. Other utilities were based on published studies conducted in other countries as a result of a paucity of utility data for the Thai DM population. All utility values are shown in Table 2.

\section{Study Outcomes}

The study estimated long-term costs, qualityadjusted life years (QALYs) which is the product of utility and life year, and clinical benefits in 
Table 3 Base-case results

\begin{tabular}{lll}
\hline & Dapagliflozin & Standard treatment \\
\hline Total cost (THB/USD) & $435,535(14,455)$ & $262,356(8707)$ \\
Total life years & 11.82 & 11.47 \\
Total QALYs & 9.58 & 9.28 \\
Incremental cost & $173,179(5748)$ & \\
Incremental life years & 0.34 & \\
Incremental QALY & 0.30 & \\
ICER (THB/LY)/(USD/LY) & $503,462(16,710)$ & \\
ICER (THB/QALY)/(USD/QALY) & $572,098(18,988)$ & \\
\hline
\end{tabular}

ICER incremental cost-effectiveness ratio, $L Y$ life year, $Q A L Y$ quality-adjusted life year

terms of heart failure hospitalization, cardiovascular death, and chronic kidney disease.

\section{Data Analyses}

\section{Base-Case Analysis}

An annual rate of $3 \%$ was used to discount future costs and outcomes in accordance with the Thai HTA guideline [29]. The incremental cost and incremental outcomes in terms of life years and QALYs were estimated. The ratio of incremental cost and incremental outcome defined as the incremental cost-effectiveness ratio was also calculated.

\section{Uncertainty Analyses}

A univariate sensitivity analysis was conducted to investigate parameter uncertainty. All variables such as transitional probabilities, costs, and utilities were varied within a specified range. If the standard deviation or standard error was available, we used it as a range for oneway sensitivity analysis. When no such data were available, costs, probabilities, and utilities were varied within $\pm 20 \%, \pm 10 \%, \pm 10 \%$ range, respectively. The results were displayed as a tornado diagram.

In addition, probabilistic sensitivity analysis was performed. A Monte Carlo simulation was run a thousand times using Microsoft Excel. The random sampling of the model parameters was based on data distribution. Beta distribution was appropriate for transitional probability and utility data because of the range of $0-1$. Gamma distribution was appropriate for cost data owing to the positive values. The results were presented as a scatter plot on the cost-effectiveness plane and a cost-effectiveness acceptability curve.

\section{Study Process}

The face validity of the model structure and input parameters was assessed by experts, such as endocrinologists, nephrologists, and cardiologists. The preliminary findings were presented in the expert meeting. All suggestions were taken into consideration.

\section{Compliance with Ethics Guidelines}

This article is based on previously conducted studies and does not contain any studies with human participants or animals performed by any of the authors.

\section{RESULTS}

\section{Base-Case Result}

As expected, add-on dapagliflozin treatment in patients with T2DM and high risk of CVD incurred higher total cost than those who 
Table 4 Clinical benefit of dapagliflozin compared with standard treatment

\begin{tabular}{llll}
\hline Clinical benefit & $\begin{array}{l}\text { Dapagliflozin } \\
\text { (per 1000 patients }\end{array}$ & $\begin{array}{c}\text { Standard treatment } \\
\text { (pear) }\end{array}$ & Difference \\
\hline Heart failure & & 146 & -12 \\
Heart failure hospitalization & 134 & 87 & -1 \\
Cardiovascular death & 86 & & -21 \\
Chronic kidney disease & & 49 & -1 \\
Macroalbuminuria & 28 & 2 & -1 \\
Dialysis & 1 & 1 & \\
Transplantation & 0 & &
\end{tabular}

received standard treatment alone $(435,535$ vs $262,356 \mathrm{THB})$, while gaining more life years (11.82 vs 11.47) and QALY (9.58 vs 9.28), yielding an incremental cost-effectiveness ratio of 503,462 THB per life year, and 572,098 THB per QALY (Table 3).

Compared with standard treatment, patients with T2DM who received add-on dapagliflozin treatment gained more clinical benefits in terms of fewer hospitalizations for heart failure, macroalbuminuria, dialysis, and transplantation (Table 4).

\section{Uncertainty Analysis Result}

The one-way sensitivity analysis showed that the cost of the medicine, the discount rate, and the utility of diabetes were the top three influential parameters (Fig. 1).

Figure 2 shows that all 1000 iterations fell in the upper right quadrant on the cost-effectiveness plane indicating that add-on dapagliflozin treatment incurred higher cost while gaining more QALYs compared with the standard treatment.

The cost-effectiveness acceptability curve represents the probability of both treatment options at different willingness to pay (WTP) levels (Fig. 3). When the level of WTP increased, dapagliflozin treatment was more likely to be a cost-effective treatment compared with standard treatment alone. At 600,000 THB, the likelihood of dapagliflozin treatment being a cost-effective strategy was about $60 \%$.

\section{DISCUSSION}

This study was the first economic evaluation of add-on dapagliflozin use in patients with T2DM and high cardiovascular risk based on efficacy data from the DECLARE-TIMI 58 study in Thailand. The estimated ICER was 572,098 THB/QALY (18,988 USD/QALY). To justify whether the new intervention is cost-effective depends on the recommended WTP in each country. According to the local threshold of 160,000 THB/QALY (5310 USD/QALY), adding dapagliflozin to this cohort population is not cost-effective.

The cost-effectiveness study conducted in the UK [30] using the data from the DECLARETIMI 58 trial reported that dapagliflozin treatment was a dominant strategy with an expected increase in QALYs and reduction in lifetime total costs compared with placebo. Although the data from the DECLARE-TIMI 58 trial was used in the two studies, the results could be different depending on several factors such as the difference in the pattern of care for similar health events, model structure, input parameters, and WTP. In this study, when the threshold of cost-effective intervention was based on the World Health Organization's recommendation at WTP of at most three times the gross domestic product (GDP), adding dapagliflozin is considered a cost-effective strategy (1 GDP per capita in Thailand is equal to 7806 USD) [31].

The efficacy inputs used in this study were based mainly on the DECLARE-TIMI 58 clinical 
$400,000 \quad 450,000 \quad 500,000 \quad 550,000 \quad 600,000 \quad 650,000 \quad 700,000 \quad 750,000$

Annual cost of dapagliflozin (11,710-17,565 THB)

Discount rate $(0-6 \%)$

Utility of diabetes $(0.733-0.895)$

Utility of stable heart failure $(0.65-0.85)$

Probability of CKD in STD (0.026-0.030)

Annual DM cost without complications (16,992-25,488 THB)

Cost of acute kidney injury (88,725-133,087 THB)

Probability of CKD in dapagliflozin (0.013-0.016)

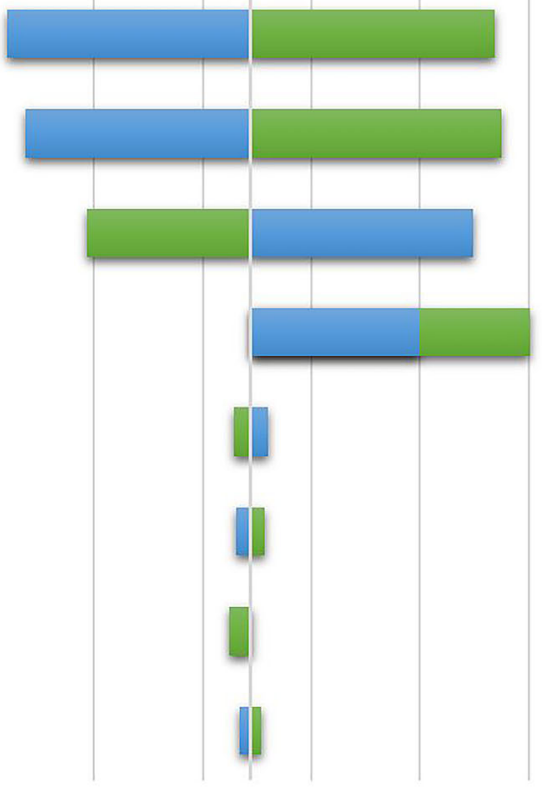

$\square$ Upper value $\quad$ Lower value

Fig. 1 Tornado diagram

trial [8], which is the largest randomized controlled trial of add-on dapagliflozin compared with the standard treatment in patients with T2DM and high cardiovascular risk. Our model was developed to capture the benefit of dapagliflozin on heart failure and renal complications reported from the trial. Several issues needed to be taken into consideration. Although the DECLARE-TIMI 58 clinical trial [8] enrolled a high cardiovascular risk population, only $10 \%$ had a history of heart failure at baseline and $6.8 \%$ had macroalbuminuria. According to the UK Prospective Diabetes Study [32], more intensive blood glucose control results in a $33 \%$ reduction in the relative risk of development of microalbuminuria or clinical grade proteinuria at 12 years. This implies that renal complication usually takes a long time for development. Within 4.2 years of the median follow-up duration in the DECLARE-TIMI 58 clinical trial, HF was found in $2.47 \%$ vs $3.33 \%$ (hazard ratio, HR 0.73; CI 0.61, 0.88) and CKD was found in $1.48 \%$ vs $2.77 \%$ (HR 0.53 ; CI 0.43 , 0.66) for dapagliflozin and standard treatment groups, respectively. The follow-up period at 4.2 years of the DECLARE-TIMI 58 study seems insufficient to determine the complication outcomes between the dapagliflozin and standard treatment groups. A longer follow-up period is required to observe a more significant reduction of complications with dapagliflozin. To address this issue, we conducted a sensitivity analysis using data from Thailand, which reported a much higher prevalence of diabetic nephropathy than the DECLARE-TIMI 58 clinical trial. The nationwide study of over 30,000 patients with T2DM collected by the National Health Security Office, Ministry of Public Health in Thailand, reported a prevalence of diabetic nephropathy of $29.7 \%$ [33]. The prevalence would be higher in tertiary care hospitals. According to the Thailand Diabetes Registry, which collected data from 9419 patients with T2DM at 11 tertiary care hospitals, the 


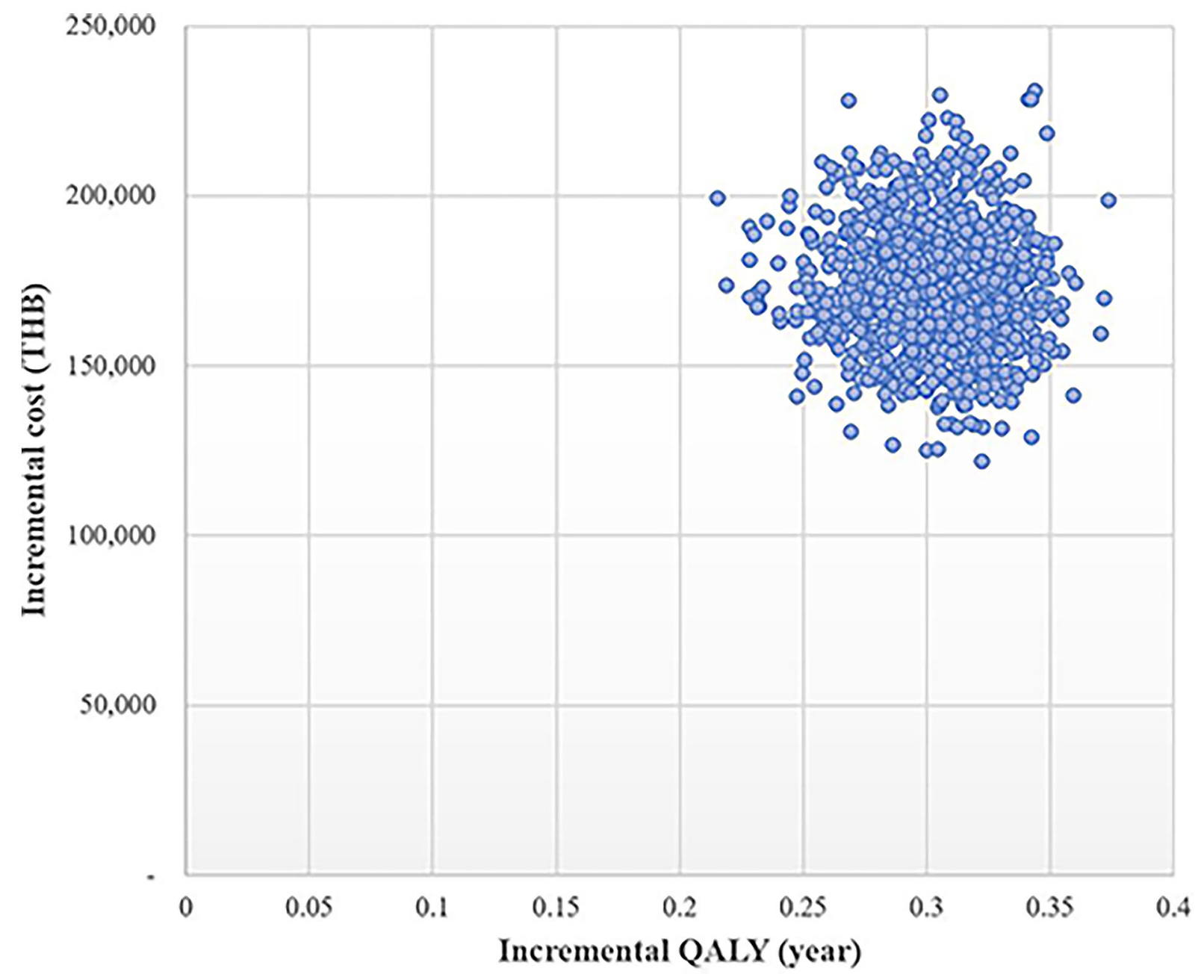

Fig. 2 Scatter plot of add-on dapagliflozin treatment compared with standard treatment on cost-effectiveness plane

prevalence of diabetic nephropathy was about $43.9 \%$ [3]. We found that the greater prevalence of diabetic nephropathy would result in lower ICER. On the basis of a prevalence of diabetic nephropathy in Thailand of 29.7-43.9\%, the estimated ICER would substantially decline to 168,466-241,474 THB/QALY (5591-8014 USD/ QALY).

Another limitation is in regard to the transitional probabilities used in the model. We derived transitional probabilities based on the median time of 4.2 years from the DECLARETIMI 58 clinical trial [8] and carried forward the constant transitional probabilities. This might not reflect the real situation that the disease might exponentially progress when time passes.
If this occurs, adding dapagliflozin would lead to a greater reduction in the cost of treatment complication.

In addition to the DECLARE-TIMI 58 clinical trial [8] which enrolled patients with T2DM and high CV risk, $10 \%$ of the population had heart failure and $6.8 \%$ had CKD. This population was different from the population in the DAPA-HF (Dapagliflozin and Prevention of Adverse Outcomes in Heart Failure) [34] and the DAPA-CKD (Dapagliflozin and Prevention of Adverse Outcomes in Chronic Kidney Disease) [35]. Both DAPA-HF and DAPA-CKD enrolled 100\% patients with $\mathrm{HF}$ and $100 \%$ patients with $\mathrm{CKD}$, respectively. The findings of the cost-effectiveness study from different populations might 


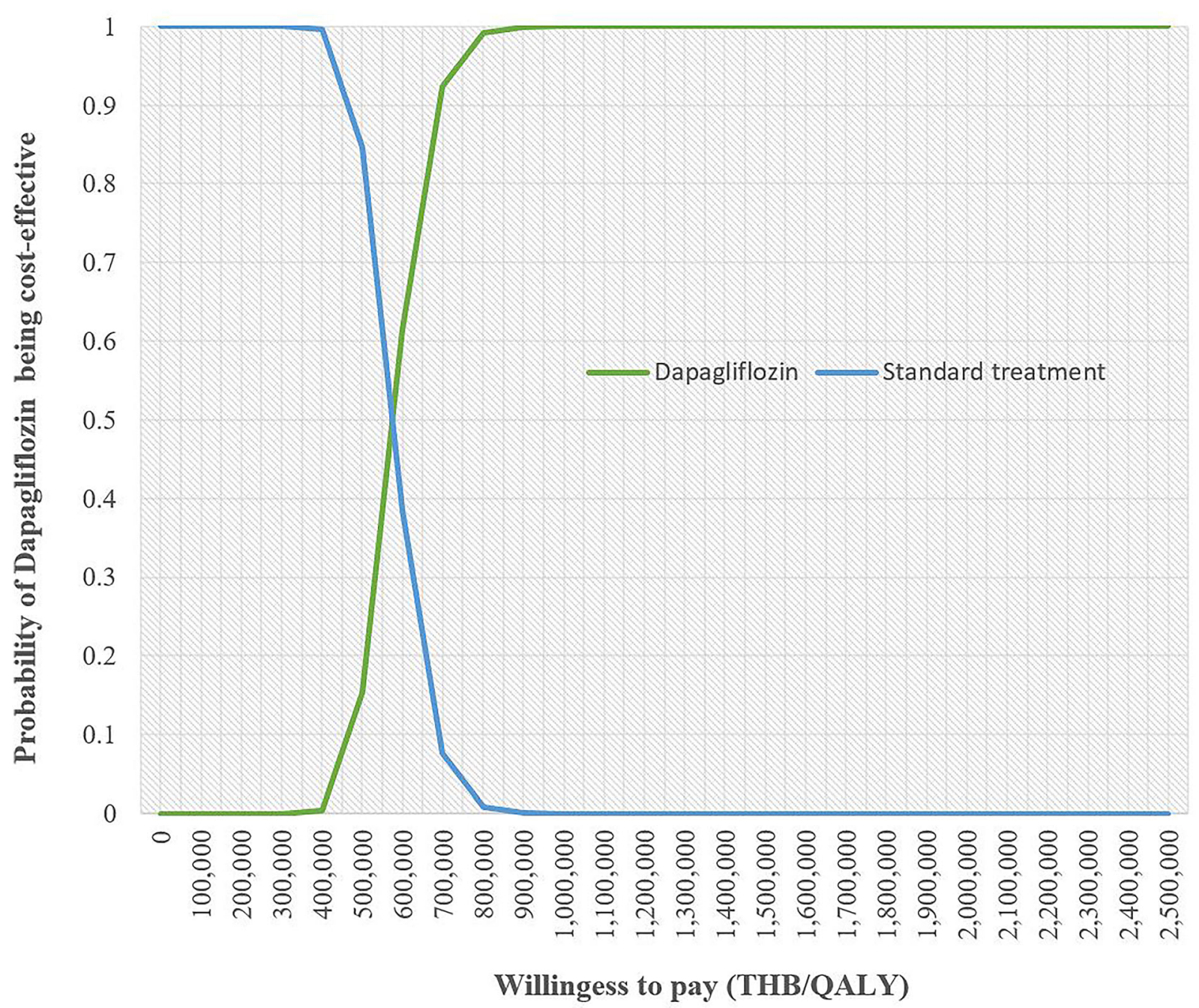

Fig. 3 Cost-effectiveness acceptability curve

show different results. In Thailand, the findings of cost-effectiveness study based on the DAPAHF trial reported that dapagliflozin is a cost-effective add-on therapy for patients with heart failure with reduced ejection fraction [36].

\section{CONCLUSIONS}

On the basis of the DECLARE-TIMI 58 study with low incidence of complications and only 4.2 years of median follow-up duration, the add-on dapagliflozin results in an ICER of 572,098 THB/QALY (18,988 USD/QALY) which exceeds the local threshold of 160,000 THB/ QALY (5310 USD/QALY). The ICER would be substantially lower in settings with high prevalence of T2DM complications.

\section{ACKNOWLEDGEMENTS}

Funding. The authors thank AstraZeneca (Thailand) for funding this study. The sponsor assisted in literature support and organized the expert meetings. However, the sponsor played no role in selecting the inputs, model structure, 
analyzing the data, or preparing the manuscript. The journal's Rapid Service Fee was also funded by AstraZeneca (Thailand).

Authorship. All named authors meet the International Committee of Medical Journal Editors (ICMJE) criteria for authorship for this article, take responsibility for the integrity of the work as a whole, and have given their approval for this version to be published.

Authors' Contributions. The following describes the contributions each author made to this work: conception and design-Chaicharn Deerochanawong, Kriengsak Vareesangthip, Dilok Piyayotai, Unchalee Permsuwan; organizing team meeting-Dittaya Thongsuk, Nuch Pojchaijongdee; input data searching-all authors; model and input data verifying-Chaicharn Deerochanawong, Kriengsak Vareesangthip, Dilok Piyayotai, Unchalee Permsuwan; data analysis-Unchalee Permsuwan; manuscript drafting-Chaicharn Deerochanawong, Unchalee Permsuwan; approval of final manuscript-all authors.

Disclosures. Chaicharn Deerochanawong, Kriengsak Vareesangthip, Dilok Piyayotai, Unchalee Permsuwan have nothing to disclose. Dittaya Thongsuk and Nuch Pojchaijongdee are current employees of AstraZeneca (Thailand) Ltd.

Compliance with Ethics Guidelines. This article is based on previously conducted studies and does not contain any studies with human participants or animals performed by any of the authors.

Data Availability. The datasets generated during and/or analyzed during the current study are available from the corresponding author on reasonable request.

Open Access. This article is licensed under a Creative Commons Attribution-NonCommercial 4.0 International License, which permits any non-commercial use, sharing, adaptation, distribution and reproduction in any medium or format, as long as you give appropriate credit to the original author(s) and the source, provide a link to the Creative Commons licence, and indicate if changes were made. The images or other third party material in this article are included in the article's Creative Commons licence, unless indicated otherwise in a credit line to the material. If material is not included in the article's Creative Commons licence and your intended use is not permitted by statutory regulation or exceeds the permitted use, you will need to obtain permission directly from the copyright holder. To view a copy of this licence, visit http:// creativecommons.org/licenses/by-nc/4.0/.

\section{REFERENCES}

1. Saeedi P, Petersohn I, Salpea P, et al. Global and regional diabetes prevalence estimates for 2019 and projections for 2030 and 2045: results from the International Diabetes Federation Diabetes Atlas, 9th edition. Diabetes Res Clin Pract. 2019;157: 107843.

2. Stratton IM, Adler AI, Neil HA, et al. Association of glycaemia with macrovascular and microvascular complications of type 2 diabetes (UKPDS 35): prospective observational study. BMJ. 2000;321(7258):405-12.

3. Rawdaree P, Ngarmukos C, Deerochanawong C, et al. Thailand diabetes registry (TDR) project: clinical status and long term vascular complications in diabetic patients. J Med Assoc Thai. 2006;89(Suppl 1):S1-9.

4. Sriphrapradang C, Thewjitcharoen Y, Buranapin S, et al. Effectiveness and safety of sodium-glucose cotransporter- 2 inhibitors in Thai adults with type 2 diabetes mellitus: a real-world study. Curr Med Res Opin. 2020;36(10):1601-10.

5. Chatterjee S, Riewpaiboon A, Piyauthakit P, et al. Cost of diabetes and its complications in Thailand: a complete picture of economic burden. Health Soc Care Community. 2011;19(3):289-98.

6. Riddle MC, Bakris G, Blonde L, Boulton AJM, D'Alessio D, DiMeglio LA. American Diabetes Association Standards of medical care in diabetes2021. Diabetes Care. 2021;44(Supplement 1): S1-232.

7. Zelniker TA, Wiviott SD, Raz I, et al. SGLT2 inhibitors for primary and secondary prevention of 
cardiovascular and renal outcomes in type 2 diabetes: a systematic review and meta-analysis of cardiovascular outcome trials. Lancet. 2019;393(10166):31-9.

8. Wiviott SD, Raz I, Bonaca MP, et al. Dapagliflozin and cardiovascular outcomes in type 2 diabetes. N Engl J Med. 2019;380(4):347-57.

9. Krittayaphong R, Permsuwan U. Cost-effectiveness analysis of sacubitril-valsartan compared with enalapril in patients with heart failure with reduced ejection fraction in Thailand. Am J Cardiovasc Drugs. 2018;18(5):405-13.

10. Sugrue DM, Ward T, Rai S, McEwan P, van Haalen HGM. Economic modelling of chronic kidney disease: a systematic literature review to inform conceptual model design. Pharmacoeconomics. 2019;37(12):1451-68.

11. Tancredi M, Rosengren A, Svensson AM, et al. Excess mortality among persons with type 2 diabetes. N Engl J Med. 2015;373(18):1720-32.

12. Health Statistics, Strategy and Planning Division, Ministry of Public Health. Public Health Statistics A. D. 2018. http://bps.moph.go.th/new_bps/sites/ default/files/statistic\%2061.pdf. Accessed 15 Sept 2020 .

13. Bundhamcharoen K, Aungkulanon S. Burden of disease in Thai population 2009. International Health Policy Program; 2012.

14. Janwanishstaporn S. National trend, in-hospital and long term outcomes in the hospitalized heart failure patients in Thailand. Thai Heart J. 2015;28: 128.

15. Krittayaphong R, Karaketklang K, Yindeengam A, Janwanishstaporn S. Heart failure mortality compared between elderly and non-elderly Thai patients. J Geriatr Cardiol. 2018;15(12):718-24.

16. Mosenzon O, Wiviott SD, Cahn A, et al. Effects of dapagliflozin on development and progression of kidney disease in patients with type 2 diabetes: an analysis from the DECLARE-TIMI 58 randomised trial. Lancet Diabetes Endocrinol. 2019;7(8): 606-17.

17. Srisubat A, Sriratanaban J, Ngamkiatphaisan S, Tungsanga K. Cost-effectiveness of annual microalbuminuria screening in Thai diabetics. Asian Biomed. 2014;8(3):371-9.

18. Adler AI, Stevens RJ, Manley SE, Bilous RW, Cull CA, Holman RR. Development and progression of nephropathy in type 2 diabetes: the United Kingdom Prospective Diabetes Study (UKPDS 64). Kidney Int. 2003;63(1):225-32.
19. Chaikledkaew U, Teerawattananon Y. Guidelines for health technology assessment in Thailand. 2nd ed. Nonthaburi: Wacharin; 2013.

20. Deerochanawong C, Ferrario A. Diabetes management in Thailand: a literature review of the burden, costs, and outcomes. Glob Health. 2013;14(9):11.

21. Riewpaiboon A, Chatterjee S, Riewpaiboon W, Piyauthakit P. Disability and cost for diabetic patients at a public district hospital in Thailand. Int J Pharm Pract. 2011;19(2):84-93.

22. Teerawattananon Y, Mugford M, Tangcharoensathien V. Economic evaluation of palliative management versus peritoneal dialysis and hemodialysis for end-stage renal disease: evidence for coverage decisions in Thailand. Value Health. 2007;10(1):61-72.

23. National Health Security Office. The administration manual for National Health Security Funding 2019. https://www.nhso.go.th/files/userfiles/file/2020/\% Е0\%В8\%АВ\%Е0\%В8\%99\%Е0\%В9\%88\%Е0\%В8\% А7\%Е0\%В8\%А2\%Е0\%В8\%9А\%Е0\%В8\%А3\%Е0\% В8\%В4\%Е0\%В8\%81\%Е0\%В8\%В2\%Е0\%В8\%А3/\% Е0\%В8\%84\%Е0\%В8\%В9\%Е0\%В9\%88\%Е0\%В8\% A1\%Е0\%B8\%B7\%E0\%B8\%AD/62_\%E0\%B8\%84\% Е0\%В8\%В9\%Е0\%В9\%88\%Е0\%В8\%А1\%Е0\%В8\% В7\%Е0\%В8\%AD\%Е0\%В8\%9A\%Е0\%В8\%А3\%Е0\% В8\%В4\%Е0\%В8\%АВ\%Е0\%В8\%В2\%Е0\%В8\%А3\% Е0\%В8\%81\%Е0\%В8\%AD\%Е0\%В8\%87\%Е0\%В8\% 97\%E0\%B8\%B8\%E0\%B8\%99\%2062.pdf. Accessed 26 Nov 2020.

24. Drug and Medical Supply Information Center. 2020. http://dmsic.moph.go.th. Accessed 24 Sept 2020.

25. Srinonprasert V, Tantai N, Maneeon S. Cost-effectiveness analysis of SGLT-2 inhibitors for treatment of patients with type 2 diabetes in Thailand. http:// www.hitap.net/documents/176100. Accessed 1 Oct 2020 .

26. Riewpaiboon A. Standard cost lists for health technology assessment. Health Intervention and Technology Assessment Program (HITAP); 2011.

27. Bureau of Trade and Economics Indices, Ministry of Commerce. CPI. 2020. http://www.price.moc.go. th/price/cpi/index_new_all.asp. Accessed 1 Oct 2020.

28. Bank of Thailand. Foreign exchange rate. 2020. https://www.bot.or.th/English/Statistics/ FinancialMarkets/ExchangeRate/_layouts/ Application/ExchangeRate/ExchangeRate.aspx. Accessed 28 Dec 2020. 
29. Permsuwan U, Guntawongwan K, Buddhawongsa P. Handling time in economic evaluation studies. J Med Assoc Thai. 2014;97(Suppl 5):S50-8.

30. McEwan P, Morgan AR, Boyce R, et al. The costeffectiveness of dapagliflozin in treating high risk patients with type 2 diabetes mellitus: an economic evaluation using data from the DECLARE-TIMI 58 trial. Diabetes Obes Metab. 2020;23:1020-9.

31. World Bank national accounts data. GDP per capital (current US\$). 2021. https://data.worldbank.org/ indicator/NY.GDP.PCAP.CD?locations=TH. Accessed 14 Jan 2021.

32. Bilous R. Microvascular disease: what does the UKPDS tell us about diabetic nephropathy? Diabet Med. 2008;25(Suppl 2):25-9.

33. Reutrakul S, Deerochanawong C. Diabetes in Thailand: status and policy. Curr Diab Rep. 2016;16(3): 28.

34. McMurray JJV, Solomon SD, Inzucchi SE, et al. Dapagliflozin in patients with heart failure and reduced ejection fraction. $\mathrm{N}$ Engl $\mathrm{J}$ Med. 2019;381(21):1995-2008.
35. Heerspink HJL, Stefánsson BV, Correa-Rotter R, et al. Dapagliflozin in patients with chronic kidney disease. N Engl J Med. 2020;383(15):1436-46.

36. Krittayaphong R, Permsuwan U. Cost-utility analysis of add-on dapagliflozin treatment in heart failure with reduced ejection fraction. Int $\mathrm{J}$ Cardiol. 2021;1(322):183-90.

37. Adena MA, Hamann G, Sindone AP. Cost-effectiveness of ivabradine in the treatment of chronic heart failure. Heart, Lung and Circ. 2019;28(3): 414-22.

38. Clarke P, Gray A, Holman R. Estimating utility values for health states of type 2 diabetic patients using the EQ-5D (UKPDS 62). Med Decis Making. 2002;22:340-9.

39. Li B, Cairns JA, Draper H, Dudley C, Forsythe JL, Johnson RJ, et al. Estimating health-state utility values in kidney transplant recipients and waitinglist patients using the EQ-5D-5L. Value Health. 2017;20(7):976-84. 